\title{
PREFACE
}

\section{Steven M. Weinreb/ Heterocycles}

Steven M. Weinreb, who is currently Russell and Mildred Marker Professor at The Pennsylvania State University, is an internationally recognized leader in the area of synthetic organic chemistry. His research has broadly encompassed both natural product total synthesis and the development of useful new synthetic methodology. Throughout his career, Weinreb has insisted on maintaining an elegant simplicity in solving problems even as his goals became increasingly more complex, His mastery of this seemingly dichotomous challenge has been a hallmark of the Weinreb oeuvre.

The Weinreb name is etched into the canon of organic chemistry due to his invention of $N$-methoxy- $N$ methyl amides in reactions with Grignards or organometallics to form ketones. In addition, his name is associated with the use of aluminum amide reagents of various types which are valuable for converting esters to amides and nitriles under mild conditions. These two methodologies have become so ensconced in the contemporary chemist's repertoire that they have now achieved generic reaction status. Of course, the emphasis of this birthday Volume is on heterocyclic materials, so the focus of our review should honor his achievements in that field.

In the arena of total synthesis of heterocyclic natural products his leadership position, sustained over the past three decades, includes several "firsts" in his early years: the initial syntheses of the interesting natural products cephalotaxine (1972), methoxatin (1981), and streptonigrin (1982). Weinreb's cephalotaxine synthesis, aside from being the first of many, is still the most efficient route to this unique alkaloid which is of potential value in cancer chemotherapy. A feature of this synthesis is an enamine acylation and a MgOMe-catalyzed Nazarov cyclization to forge the cyclopentenyl ring. The streptonigrin project utilized an imino Diels-Alder reaction as a key step and prefigured the future development of groundbreaking hetero Diels-Alder methodology in the Weinreb lab. Also featured in the synthesis was the brilliant exploitation of the classic pyridine- $N$-oxide rearrangement for functionalization of the pyridine ring along with a sophisiticated 2,3-sigmatropic rearrangement to migrate functionality from the 2 to the 3 position of the pyridine. This synthesis is a tour de force of creative heterocyclic chemistry. Recently a new general enantioselective approach to the Securinega class of CNS-active plant alkaloids has been developed by his group, and has been applied to the first total synthesis of 
phyllanthine (1999). Most recently, the first synthesis of ageladine A, a novel imidazopyridine, which features Suzuki chemistry and a novel aza-electrocyclization, has been reported (2006).

Other major total synthesis achievements include some molecules for which Weinreb reported not the first, but what are undoubtedly the best, most elegant syntheses to date. He has reported an exceptionally direct total synthesis of the marine alkaloid papuamine (1994) utilizing a novel, previously unknown type of imino ene pericyclic reaction and this same methodology was also applied to synthesis of the Amaryllidaceae alkaloids coccinine and pancracine (1997).

Weinreb has pioneered the development and application of hetero Diels-Alder chemistry in organic synthesis, and coauthored (with Dale Boger) a widely cited book on this subject. He has extensively utilized intramolecular imino Diels-Alder reactions in alkaloid total synthesis, including targets such as $\delta$ coniceine (1979), tylophorine (1979), cryptopleurine (1983), elaeokanine A (1980), slaframine (1982), anhydrocannibisativine (1984) and epi-lupinine (1983). He has clearly shown that cycloadditions using imino dienophiles are valuable in synthesis of diverse types of heterocycles. Many of his accomplishments in the general area of alkaloid total synthesis have become benchmarks by which progress in the field is measured.

In addition, the Weinreb group has expanded this interest in hetero Diels-Alder chemistry to include the versatile $N$-sulfinyl dienophiles. Weinreb elegantly demonstrated that this once obscure reaction has exceptional potential for stereospecific synthesis of unsaturated vicinal amino alcohols and vicinal diamines. The $N$-sulfinyl Diels-Alder cycloadducts are key intermediates intermediates which are then subjected to sigmatropic rearrangements for further elaboration. This methodology has been cleverly applied to preparation of some amino sugars and to threo- and erythro-sphingosine using both inter and intramolecular versions of the cycloaddition. He has also utilized $N$-sulfinyl Diels-Alder cycloadducts in short, efficient syntheses of the amino sugar moieties of the antitumor agent nogalamycin and of the interesting protein kinase $\mathrm{C}$ inhibitor staurosporine.

Another aspect of hetero Diels-Alder methodology which has been explored by Weinreb and coworkers involves azadienes. He has shown that intramolecular cyclizations of oxazoles with olefins can be used to prepare annulated pyridines and ingeniously applied this strategy to the first total synthesis of the unique alkaloid eupolauramine (1983). Weinreb reported studies on intramolecular cyclizations of $N$ - 
acylimines with alkenes to afford annulated dihydrooxazines. This methodology has great potential in alkaloid and $\alpha$-amino acid synthesis, and has been applied in a stereospecific approach to the amino acid component of the antibiotic nikkomycin (1988).

More recently, he has demonstrated the generality of the Kresze reaction of aldehydes with $N$-sulfinyl compounds to prepare electron deficient imines and has utilized these species in a variety of useful synthetic processes. For example, $N$-sulfonyl imines can be prepared easily and converted to a number of amine derivatives. Reactions of these $N$-sulfonyl imines with olefins and vinyl silanes have also been explored and applied in a synthesis of the alkaloid (+)-lycoricidine (1993).

An important aspect of the Weinreb lab contribution to the field of heterocyclic natural products is the use of total synthesis to correct structural assignment. A brilliant example of this is his study of cylindrospermopsin. The work included two stereospecific syntheses, the first showing that the assigned structure was incorrect. Then, use of the same methods, which involved an intramolecular sulfinylurea Diels-Alder followed by sigmatropic rearrangement, a new uracil synthesis and a cyclic guanidinium construction, culminated in a structure proof. A second important example of "disproof / proof" is his research on lepadiformine. In addition to the proof of structure by synthesis, Weinreb also used a second synthetic scheme to establish the absolute configuration of this important natural product.

A short overview of some important Weinreb research that is not specifically heterocyclic is in order. He has introduced practical new nitrogen protecting groups ( $\beta$-trimethylsilylethanesulfonyl=SES, tertbutylsulfonyl=Bus and $\beta$-tosylethyl=TSE) and the reagent TsNHBoc has been recently developed for use in Mitsonobu reactions. He has also devised novel methodology for the construction of polycyclic ring systems. For example, he discovered an efficient new annulation reaction (the "Weinreb-Staunton" annulation) which provides anthracycline systems in one of the most efficient assembly strategies to date. In fact, this protocol was used as a key step in the Weinreb olivin total synthesis.

Other successes include an efficient stereoselective route to the antitumor antibiotic actinobolin (1985) and the successful extention of the basic strategy to the first total synthesis of the congeneric molecule bactobolin (1988). Both syntheses proved to be excellent showcases for some of the clever synthetic methodology which he developed such as the Weinreb ketone synthesis and the use of aluminum amides for converting esters to amides. 
With the recent Arthur C. Cope Senior Scholar award crowning his list of honors, lectureships, and consultancies, it is clear that the community of organic chemists recognizes and appreciates Steve Weinreb. He has sustained a highly productive research program that has contributed to the practice of organic chemistry through both significant new synthetic protocols as well as total syntheses of a number of important natural products. This body of work exemplifies his innovative and deft touch in practicing the art of organic synthesis.

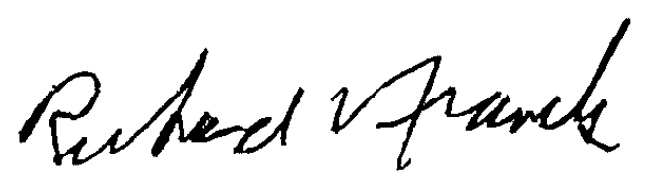

Richard W. Franck

Hunter College of CUNY 